\title{
ADAPTIVE BIT PARTITIONING STRATEGY FOR CELL-EDGE USERS IN MULTI-ANTENNA MULTICELL NETWORKS
}

\author{
Berna Ö̈bek ${ }^{1,2}$, Didier Le Ruyet ${ }^{1}$ \\ ${ }^{1}$ LAETITIA/CEDRIC, CNAM, Paris, France \\ ${ }^{2}$ Electrical and Electronics Engineering Dept., Izmir Institute of Technology, Izmir, Turkey \\ berna.ozbek@cnam.fr,didier.le_ruyet@cnam.fr
}

\begin{abstract}
In this paper, we improve the performance of cell-edge users by applying adaptive bit partitioning for intercell interference cancelation in cooperative downlink multicell systems. The strategy for partitioning quantization bits between serving and interfering base stations affects the performance of the cooperative networks which is quite sensitive to the quality of the channel state information. The existing transmission strategies are based on the maximization of average cell rate which results in a reduced rate for the users in the cell-edge regions. We propose an adaptive bit partitioning strategy to improve the rate of cell-edge users at the same average feedback link rate. We show the performance of the cell-edge users is significantly increased while keeping the same average cell rate.
\end{abstract}

\section{INTRODUCTION}

In next generation wireless networks, the frequency reuse factor is set to one to increase the spectral efficiency which causes intercell interference (ICI). In order to eliminate the interference coming from the other cells, coordinated transmission strategies are employed in multi-antenna multicell networks by exchanging users' channel state information (CSI) among base stations (BS)s [1]. The use of multiple antenna techniques in downlink wireless networks increases the overall throughput by exploiting the degrees of freedom in the spatial domain. It is possible to accommodate up to $N_{t}-1$ interference signals in a coordinated multicell transmission when a base station is equipped with $N_{t}$ antennas by using linear transceiver processing techniques.

A full coordinated multicell transmission requires to exchange all the users' CSI as well as their data information. In order to reduce the backhaul load, partial cooperative strategies have been considered where the BSs share only the users' CSIs [2]. In partial coordinated multicell networks, each base station designs its beamforming vector for communicating its own user by employing different transmission strategies such as Maximum Ratio Combining (MRC) and Partial ZeroForcing (PZF) [3]. It is important to develop cooperative techniques with limited feedback that maximize the performance while keeping the backhaul load at a reasonable level.

The performance of cooperative multicell networks is highly dependent on the quality of the CSIs from both serving and interfering BSs which are quantized and fed back by the users through a limited feedback link. The strategy of bit partitioning between the serving and interfering BSs affects the overall performance of cooperative networks. Adaptive limited feedback schemes are presented in [4][5] by selecting the transmission strategy according to users' locations and the power level at cell edge. With a constraint on the total number of feedback bits for each user, the adaptive bit partitioning is performed to maximize the sum rate and consequently the transmission strategy is assigned. It is shown that bit partitioning between the serving and interfering BSs only depends on the location of the user itself rather than the location of the other users in the interfering cells.

All these strategies have assumed that each user has a fixed number of bits to feedback the CSIs of the serving and interfering BSs without taking into account its position in the cell. The partitioning of the quantization bits between the serving and interfering BSs can improve the overall capacity while providing poor performance for cell-edge users. In [6], an efficient method called precoding matrix index (PMI) restriction is introduced to mitigate ICI for cell-edge users by informing the other BSs about the precoding vector which causes large interference. In this paper, we propose an adaptive bit partitioning strategy to improve the performance of cell-edge users while keeping the same average rate on feedback link. This method is based on allocating more bits to quantize CSI of the users far from the BS to efficiently perform ICI cancelation.

This paper is organized as follows. In Section II, the system model for multi-antenna multicell networks based on the Wyner model is described including the limited feedback transmission strategies. In Section III, we present the proposed strategy to improve the performance of cell-edge users in limited feedback link including analytical derivations for optimum bit partitioning. In Section IV and V, the performance results are illustrated and the concluding remarks are drawn respectively. 


\section{SYSTEM MODEL}

As shown in Fig. 1, a downlink multicell wireless network based on the Wiener model is considered where the base station in each cell is composed of $N_{t}$ transmit antennas and each user is equipped with a single antenna.

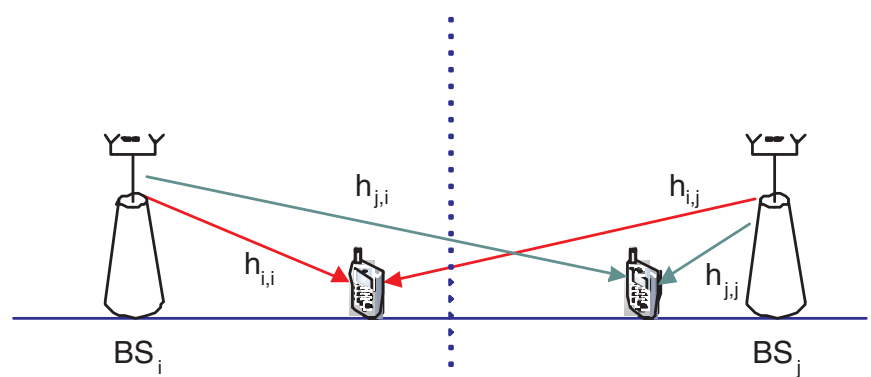

Fig. 1. A wiener multicell system with $N_{t}$ transmit antennas at each cell

Using a narrow band flat-fading model, the baseband received signal for the user in cell $i$ is written as,

$$
y_{i}=\sqrt{P_{i, i}} \mathbf{h}_{i, i}^{H} \mathbf{w}_{i} x_{i}+\sum_{j \neq i} \sqrt{P_{i, j}} \mathbf{h}_{i, j}^{H} \mathbf{w}_{j} x_{j}+n_{i}
$$

where $P_{i, j}$ is the received power of user $i$ from BS $j, \mathbf{h}_{i, j}$ is the channel between the user of cell $i$ and BS $j . \mathbf{w}_{i}$ is the beamforming vector at $\mathrm{BS} i$ and $n_{i}$ is the complex additive white Gaussian noise at the receive antenna with zero mean and $\mathbf{E}\left\{\left|n_{i}\right|^{2}\right\}=1$. It is assumed that each component of $\mathbf{h}_{i, j}$ has independent identically distributed random variable with $\mathcal{C N}(0,1)$ and $\mathbf{E}\left\{\left\|x_{i}\right\|^{2}\right\}$ is normalized to 1 .

The associated average rate of each user in cell $i$ is calculated as,

$$
R_{i}=\mathbb{E}\left[\log _{2}\left(1+\mathrm{SINR}_{i}\right)\right]
$$

where the instantaneous SINR of the user in cell $i$ is:

$$
\operatorname{SINR}_{i}=\frac{P_{i, i}\left|\mathbf{h}_{i, i}^{H} \mathbf{w}_{i}\right|^{2}}{1+\sum_{j \neq i} P_{i, j}\left|\mathbf{h}_{i, j}^{H} \mathbf{w}_{j}\right|^{2}}
$$

The received power from serving and interfering BSs are calculated considering the following path loss model :

$$
P_{i, j}=P_{0}\left(\frac{d_{i, j}}{R}\right)^{-\alpha}
$$

with $R$ is the radius of the cell, $P_{0}$ is the received power at the cell edge, $\alpha$ is the path loss exponent and $d_{i, j}$ is the distance between user $i$ and BS $j$.

\subsection{Limited Feedback Channel}

It is assumed that each user has a perfect knowledge of CSI belonging to serving and interfering BSs and the channel quality information (CQI) is perfectly available at the transmitter side. The users feed back their channel direction information (CDI) to the BS associated to all these links.

Before sending back channel information, the user quantizes the direction of the channel vectors, $\tilde{\mathbf{h}}_{i, j}=\frac{\mathbf{h}_{i, j}}{\left\|\mathbf{h}_{i, j}\right\|}$, according to the available partitioning bits by exploiting a quantization codebook $\mathfrak{C}=\left\{\mathbf{c}_{i, j}^{1}, \mathbf{c}_{i, j}^{2}, \ldots, \mathbf{c}_{i, j}^{M}\right\}$ where $M=2^{B_{i, j}}$ is the number of codewords and $B_{i, j}$ is the number of quantization bits. The codebook composed of $N_{t}$ dimensional unit vectors is designed using by random vector quantizer (RVQ) [7]. By using the minimum chordal distance metric, the indices of CDI for both the serving and interfering channel vectors are obtained as,

$$
\begin{aligned}
n_{j} & =\arg \min _{1 \leq m \leq M} 1-\left|\left(\mathbf{c}_{i, j}^{m}\right)^{H} \tilde{\mathbf{h}}_{i, j}\right|^{2} \\
\hat{\mathbf{h}}_{i, j} & =\mathbf{c}_{i, j}^{n_{j}}
\end{aligned}
$$

\subsection{Transmission Strategies}

In this work, two classical transmission strategies are considered while designing the beamforming vectors $\mathbf{w}_{i}$.

\section{1) Maximum Ratio Combining (MRC):}

The users in the other cells are far from the BS and no interference cancellation is needed. Therefore, the precoding vector is designed according to the channel direction of the serving user. For BS $i$, the precoding vector is determined by,

$$
\hat{\mathbf{w}}_{i}=\hat{\mathbf{h}}_{i, i}
$$

The distribution of the received power is $2\left|\mathbf{h}_{i, i}^{H} \hat{\mathbf{w}}_{i}\right|^{2} \sim \chi_{2 N_{t}}^{2}$ where $\chi_{n}^{2}$ denotes the chi-square random variable with $n$ degrees of freedom.

\section{2) Partial Zero-Forcing (PZF):}

In order to maximize $\left|\mathbf{h}_{i, i}^{H} \mathbf{w}_{i}\right|^{2}$, some degrees of freedom are used for ICI cancelation. This corresponds to selecting $\mathbf{w}_{i}$ in the direction of the projection of the channel vector $\hat{\mathbf{h}}_{i, i}$ on the nullspace of $\hat{\mathbf{H}}_{i}=\left[\hat{\mathbf{h}}_{2, i}, \ldots, \hat{\mathbf{h}}_{i-1, i}, \hat{\mathbf{h}}_{i+1, i}, \ldots, \hat{\mathbf{h}}_{K, i}\right]$. The precoding vector is given by

$$
\mathbf{w}_{i}^{\prime}=(\mathbf{I}-\mathbf{P}) \hat{\mathbf{h}}_{i, i}
$$

where $\mathbf{P}=\hat{\mathbf{H}}_{i}\left(\hat{\mathbf{H}}_{i}^{*} \hat{\mathbf{H}}_{i}\right)^{-1} \hat{\mathbf{H}}_{i}^{*}$ which is the projection matrix on $\hat{\mathbf{H}}_{i}$.

Then, the precoding vector is obtained by applying normalization as $: \hat{\mathbf{w}}_{i}=\mathbf{w}_{i}^{\prime} /\left\|\mathbf{w}_{i}^{\prime}\right\|$.

For PZF precoding, the distribution of the received power [3] is $2\left|\mathbf{h}_{i, i}^{H} \hat{\mathbf{w}}_{i}\right|^{2} \sim \chi_{2\left(N_{t}-(K-1)\right)}^{2}$.

\subsection{Selection of transmission strategies}

We propose a strategy for multi-antenna multicell networks to increase the performance of the cell-edge users in limited feedback link. The cells are divided in two regions: the cooperative region corresponding to the edge of the cell and the non cooperative region corresponding to the center of the cell. 
$\mathrm{BS}_{\mathrm{i}}$ will perform PZF strategy if the user in cell $j$ is in the cooperative region. In that case, user $j$ will feedback $\hat{\mathbf{h}}_{j, i}$ to $\mathrm{BS}_{\mathrm{i}}$ through limited feedback channel. On the other hand, $\mathrm{BS}_{\mathrm{i}}$ will perform MRC strategy if the user in cell $j$ is in the non cooperative region.

Assuming that the user $j$ is in the cooperative region, the average rate of user $i$ is calculated as [4][5],

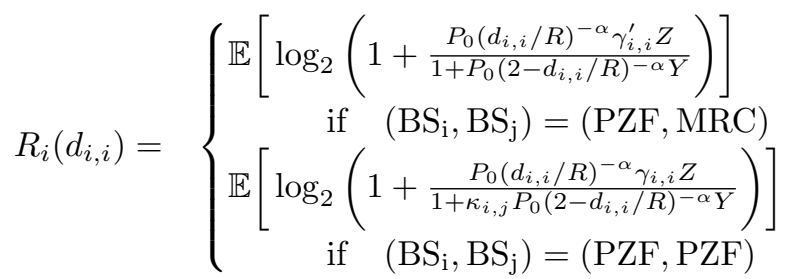

where $\gamma_{i, i}, \gamma_{i, i}^{\prime}$ and $\kappa_{i, j}$ are the quantization errors and $2 Z \sim$ $\chi_{2\left(N_{t}-1\right)}^{2}$ and $2 Y \sim \chi_{2}^{2}$.

Since RVQ is used, $\gamma_{i, i}, \gamma_{i, i}^{\prime}$ and $\kappa_{i, j}$ are calculated as follows [7] [8]:

$$
\begin{aligned}
& \gamma_{i, i}=1-2^{B_{i, i}} \beta\left(2^{B_{i, i}}, \frac{N_{t}}{N_{t}-1}\right) \\
& \gamma_{i, i}^{\prime}=1-2^{B_{i, i}^{\prime}} \beta\left(2^{B_{i, i}}, \frac{N_{t}}{N_{t}-1}\right)
\end{aligned}
$$

and

$$
\kappa_{i, j}=2^{B_{i, j}} \beta\left(2^{B_{i, j}}, \frac{N_{t}}{N_{t}-1}\right)
$$

where $\beta(x, y)$ is the Beta function, $B_{i, i}$ and $B_{i, j}$ are the numbers of bits used for the quantization of the normalized channel vector $\tilde{\mathbf{h}}_{i, i}$ and $\tilde{\mathbf{h}}_{i, j}$ in the cooperative region respectively. In the non cooperative region, $B_{i, i}$ is replaced by $B_{i, i}^{\prime}$ to quantize the CDI of the serving BS.

\section{PROPOSED BIT PARTITIONING}

The purpose of the proposed bit partitioning is to maximize the rate of cell-edge users while keeping the same average feedback rate. For user $i$, depending on its location, an ICI cancelation is performed or not by the other base station. In order to determine the $B_{i, i}^{\prime}, B_{i, i}, B_{i, j}$ values, we propose to calculate the user rate at two extreme locations. The first location is the border between the non-cooperative and the cooperative region. The second one is the cell-edge where the user requires ICI cancelation. For a given normalized distance $d_{t h}=d_{i, i} / R$ between the serving BS and the user, it is possible to improve the rate of the users within that region which is between $d_{t h}$ and 1 .

Assuming that MRC is performed by BS $i$ for user $j$, the rate at these two extreme positions are defined as $R_{i, b s}$ and
$R_{i, c e l l}$ respectively:

$$
\begin{aligned}
R_{i, b s} & =\mathbb{E}\left[\log _{2}\left(1+\frac{P_{0} d_{t h}^{-\alpha} \gamma_{i, i}^{\prime} Z}{1+P_{0}\left(2-d_{t h}\right)^{-\alpha} Y}\right)\right] \\
R_{i, \text { cell }} & =\mathbb{E}\left[\log _{2}\left(1+\frac{P_{0} \gamma_{i, i} Z}{1+\kappa_{i, j} P_{0} Y}\right)\right]
\end{aligned}
$$

where $2 Z \sim \chi_{2 N_{t}}^{2}$ and $2 Y \sim \chi_{2}^{2}$.

Our criterion is based on the maximization of the sum of the rate at these two extreme locations to improve the rate of cell-edge users under the constraint that the average number of feedback bits within each cell is fixed.

The optimization problem is defined by:

$$
\max _{B_{i, i}^{\prime}, B_{i, i}, B_{i, j}} R_{w}=R_{i, b s}+R_{i, \text { cell }}
$$

under the constraints:

$$
\begin{aligned}
d_{t h} B_{i, i}^{\prime}+\left(1-d_{t h}\right)\left(B_{i, i}+B_{i, j}\right)=B_{\text {avg }} \\
B_{i, i}^{\prime}>0 \\
B_{i, i}>0 \\
B_{i, j}>0
\end{aligned}
$$

The first constraint means that the average number of feedback bits within each cell remains fixed for Wiener model. The other constraints imply that $\tilde{\mathbf{h}}_{i, i}$ and $\tilde{\mathbf{h}}_{i, j}$ (cooperative region) and $\tilde{\mathbf{h}}_{i, i}$ (non cooperative region) are quantized with at least one bit.

To simplify the analysis, the upper bound with the mean loss in sum rate is written as,

$$
\begin{aligned}
R_{w} & \geq \mathbb{E}\left[\log _{2}\left(\frac{\gamma_{i, i}^{\prime} P_{0} d_{t h}^{-\alpha} Z}{1+P_{0}\left(2-d_{t h}\right)^{-\alpha} Y}\right)\right] \\
& +\mathbb{E}\left[\log _{2}\left(\frac{\gamma_{i, i} P_{0} Z}{1+\kappa_{i, j} P_{0} Y}\right)\right]
\end{aligned}
$$

By using some properties of expectation, we obtain:

$$
\begin{aligned}
R_{w} & \geq \log _{2}\left(\gamma_{i, i}^{\prime} P_{0} d_{t h}^{-\alpha}\right)+\log _{2}(\mathbb{E}[Z]) \\
& -\log _{2}\left(1+P_{0}\left(2-d_{t h}\right)^{-\alpha} \mathbb{E}[Y]\right) \\
& \left.+\log _{2}\left(\gamma_{i, i} P_{0}\right)+\log _{2}(\mathbb{E}[Z])-\log _{2}\left(1+\kappa_{i, j} P_{0}\right) \mathbb{E}[Y]\right)
\end{aligned}
$$

Since $\mathbb{E}[Z]=N_{t}$ and $\mathbb{E}[Y]=1$ are constant values and the quantization error is upper bounded by $\mathbb{E}\left[\sin ^{2}\left(\theta_{i, j}\right)\right]<$ $2^{-\frac{B_{i, j}}{N_{t}-1}}$ where $\theta$ is the angle between the real and quantized channel direction, it is rewritten as,

$$
\begin{aligned}
R_{w} & \geq \log _{2}\left(\left(1-2^{-\frac{B_{i, i}^{\prime}}{N_{t}-1}}\right) P_{0} d_{t h}^{-\alpha}\right) \\
& -\log _{2}\left(1+P_{0}\left(2-d_{t h}\right)^{-\alpha}\right) \\
& +\log _{2}\left(\left(1-2^{-\frac{B_{i, i}}{N_{t}-1}}\right) P_{0}\right)-\log _{2}\left(1+2^{-\frac{B_{i, j}}{N_{t}-1}} P_{0}\right) \\
& -\log _{2}\left(1+2^{-\frac{B_{i, j}}{N_{t}-1}} P_{0}\right) \\
& =R_{w p}
\end{aligned}
$$


Then, the values of $B_{i, i}^{\prime}, B_{i, i}, B_{i, j}$ that maximize the bound of the sum rate are obtained using Lagrange multipliers.

$$
\begin{aligned}
L\left(B_{i, i}^{\prime}, B_{i, i}, B_{i, j}, \lambda\right) & =R_{w p}+\lambda d_{t h} B_{i, i}^{\prime} \\
& +\lambda\left(1-d_{t h}\right)\left(B_{i, i}+B_{i, j}\right)-\lambda B_{\text {avg }}
\end{aligned}
$$

Then, we have:

$$
\begin{aligned}
\frac{\partial L}{\partial B_{i, i}^{\prime}} & =\frac{1}{\left(N_{t}-1\right)} \frac{2^{-B_{i, i}^{\prime} /\left(N_{t}-1\right)}}{\left[1-2^{-B_{i, j}^{\prime} /\left(N_{t}-1\right)}\right]}+\lambda d_{t h}=0 \\
\frac{\partial L}{\partial B_{i, i}} & =\frac{1}{\left(N_{t}-1\right)} \frac{2^{-B_{i, i} /\left(N_{t}-1\right)}}{\left[1-2^{-B_{i, i} /\left(N_{t}-1\right)}\right]}+\lambda\left(1-d_{t h}\right)=0 \\
\frac{\partial L}{\partial B_{i, j}} & =\frac{P_{0}}{\left(N_{t}-1\right)} \frac{2^{-B_{i, j} /\left(N_{t}-1\right)}}{\left[1+P_{0} 2^{-B_{i, j} /\left(N_{t}-1\right)}\right]}+\lambda\left(1-d_{t h}\right)=0
\end{aligned}
$$

By solving the equations, we obtain:

$$
\begin{aligned}
B_{i, i}^{\prime} & =\left(1-N_{t}\right) \log _{2}\left(\frac{\lambda A}{\lambda A-1}\right) \\
B_{i, i} & =\left(1-N_{t}\right) \log _{2}\left(\frac{-\lambda B}{1-\lambda B}\right) \\
B_{i, j} & =\left(1-N_{t}\right) \log _{2}\left(\frac{-\lambda B}{P_{0}(1+\lambda B)}\right)
\end{aligned}
$$

where $A=d_{t h}\left(N_{t}-1\right)$ and $B=\left(1-d_{t h}\right)\left(N_{t}-1\right)$.

Since the obtained values $B_{i, i}^{\prime}, B_{i, i}, B_{i, j}$ are positive real numbers, a round operation is performed to get integer values.

$\lambda$ can be calculated for given $d_{t h}, P_{0}, B_{\text {avg }}, N_{t}$ by solving the following equation:

$$
\left(\frac{\lambda A}{A-1}\right)^{A}\left(\frac{(\lambda B)^{2}}{P_{0}\left(1-(\lambda B)^{2}\right)}\right)^{B}-2^{B_{\text {avg }}}=0
$$

\section{PERFORMANCE RESULTS}

We illustrate the performance results to show the benefits of the proposed strategy in a cooperative multicell system. For the simulations, the parameters are chosen as $N_{t}=4, P_{0}=$ $10 \mathrm{~dB}, R=1 \mathrm{~km}$ and $\alpha=3.7$.

Firstly, we compare the performance results for the case where the number of feedback bits is fixed for each user. The equal sharing means that in the non cooperative region, $B_{a v g}$ bits are used to quantize the CDI of the serving BS and in the cooperative region, $B_{\text {avg }} / 2$ bits are used to quantize the CDIs of the serving and interfering BS. As it is shown in Fig. 4, the proposed strategy with adaptive bit partitioning increases the user rate significantly in the cell-edge region while the average feedback rate remains the same.

As illustrated in Fig. 3, the average sum cell rate $\left(R_{i}+R_{j}\right)$ is not degraded when using the proposed strategy at different average feedback rates for $d_{t h}$ varying between 0.6 and 0.8 .

In Fig. 4, the average rate of user $i$ at the cell edge region is shown considering different numbers of average feedback

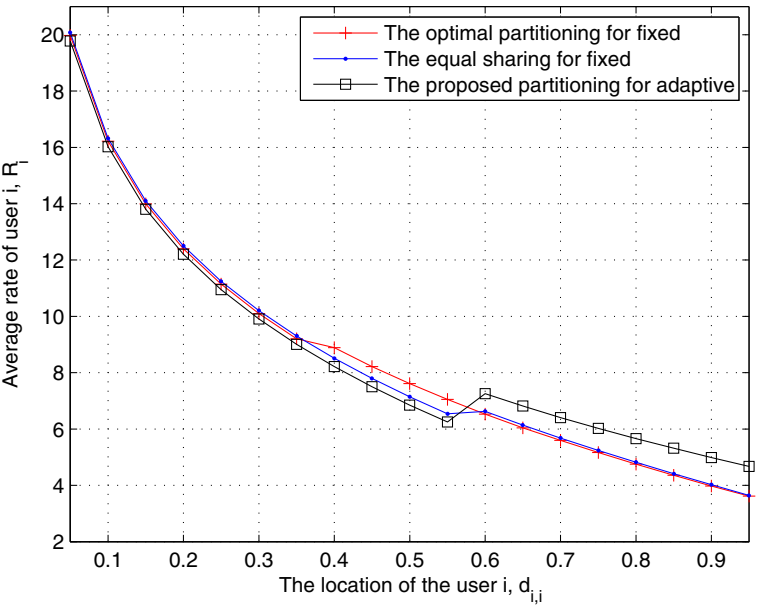

Fig. 2. The average rate of user $i$ at different locations in the cell for $d_{t h}=0.6$ and $B_{\text {avg }}=12$.

bits and assuming that the position of user $j$ is uniformly distributed in its own cell. The proposed adaptive bit partitioning improves the user rate in the cell-edge significantly compared to a fixed bit partitioning scheme while having the same feedback load. When the feedback rate increases, it is shown that the performance of the cell-edge users also increases.

The performance of the proposed strategy is also compared with PMI restriction scheme for $d_{t h}=0.6$ and $B_{a v g}=$ 7. In order to obtain the same average feedback link, the number of quantization bits is chosen as $B_{i, i}^{\prime}=B_{i, i}=B_{i, j}=5$ for both serving and interfering BSs in PMI restriction method. For the proposed algorithm, the number of quantization bits is selected as $B_{i, i}^{\prime}=3, B_{i, i}=4, B_{i, j}=9$. According to Monte-Carlo simulation results shown in Fig. 4, the proposed bit partitioning scheme gives better performance at the celledge region than PMI restriction scheme.

\section{CONCLUSION}

In this paper, we have considered adaptive bit partitioning strategy to improve the cell-edge users' rate in cooperative multi-antenna multicell networks. The CDI of serving and interfering BSs for the users in the cell-edge are quantized precisely to reduce the interference effect due to the other cell. The analytical derivations are presented for the optimum bit partitioning scheme for cell-edge users under the constraint that the average feedback rate is constant. The proposed strategy improves the cell-edge users' capacity up to $50 \%$ by only reducing $10 \%$ of the performance of the users at the non cooperative region. In addition to that, the proposed strategy achieves better performance than PMI restriction scheme at the cell edge region while keeping the same average sum cell rate and the feedback rate. 


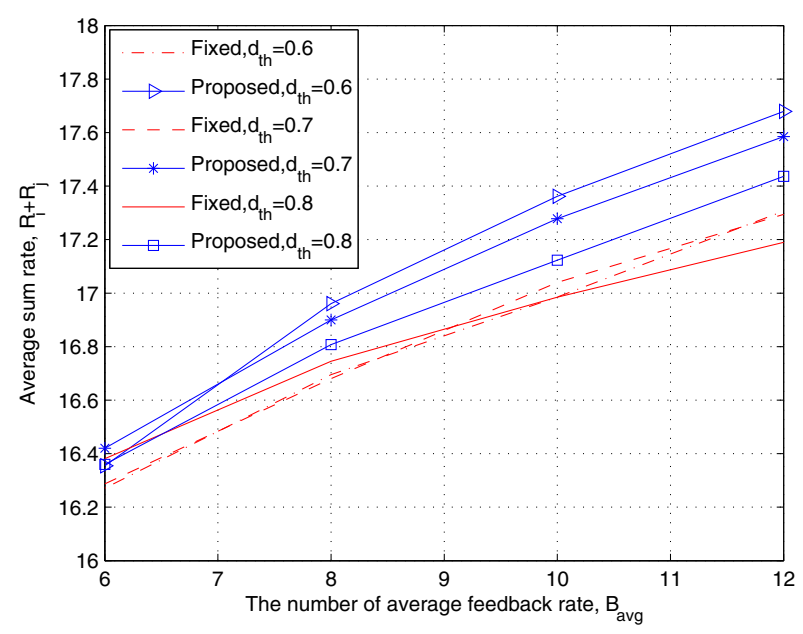

Fig. 3. The average sum cell rate at different feedback rates.

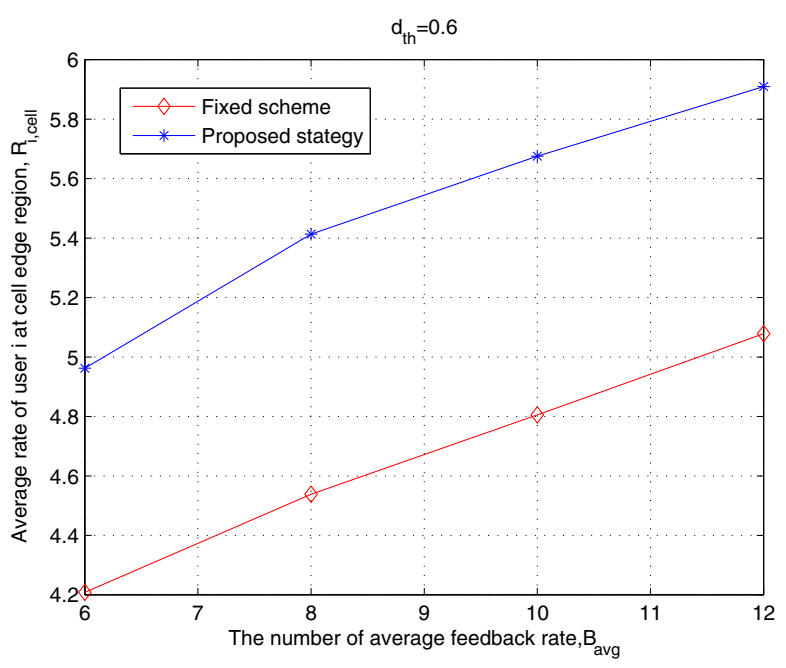

Fig. 4. The average rate of user $i$ in cell-edge region.

\section{Acknowledgment}

This research was supported by a Marie Curie Intra European Fellowship within the 7th European Community Framework Programme as a part of INTERCELL project with the contract number PIEF-GA-2009-255128.

\section{REFERENCES}

[1] H. Huang S. Shamai D. Gesbert, S. Hanly and O. Simeone, "Multi-cell mimo cooperative networks: A new look at interference," IEEE Journal on Selected Areas in Communications, vol. 28, pp. 1-29, December 2010.

[2] F. Boccardi and H. Huang, "Limited downlink network coordination in cellular networks," IEEE Int. Symp. on

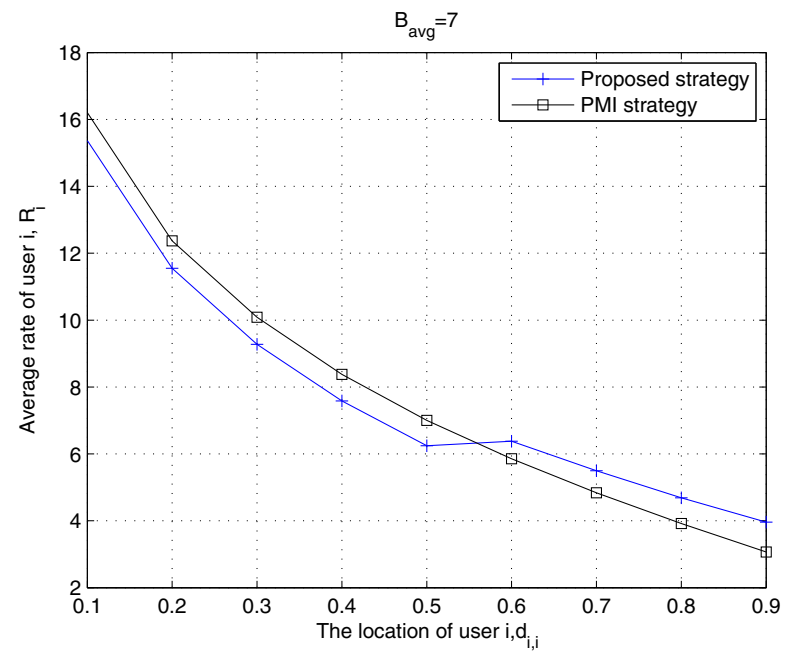

Fig. 5. The comparison of the average rate of user $i$ at different locations in the cell.

Personal Indoor and Mobile Radio Comm. (PIMRC), 2007.

[3] J. G. Andrews N. Jindal and S. Weber, "Rethinking mimo for wireless networks: Linear throughput increases with multiple receive antennas," IEEE Intl. Conf. on Communications (ICC), 2010.

[4] J. Zhang and J. G. Andrews, "Adaptive spatial intercell interference cancellation in multicell wireless network," IEEE Journal on Selected Areas in Communications, vol. 28, pp. 1455-1468, December 2010.

[5] B. Ozbek and D. Le Ruyet, "Adaptive limited feedback for intercell interference cancelation in cooperative downlink multicell networks," IEEE 7th International Symposium on Wireless Communication Systems (ISWCS), 2010.

[6] J. Lim D. Kim, S. Kim and B.-C. Ihm, "Pmi restriction for the downlink closed-loop mimo," IEEE Std, IEEEC802.16m-08/430, 2008.

[7] C. K. Au-Yeung and D. J. Love, "Performance analysis of random vector quantization limited feedback beamforming," IEEE Asilomar Conf. on Signals, Systems, and Computers, 2005.

[8] N. Jindal, "Mimo broadcast channels with finite-rate feedback," IEEE Transactions on Information Theory, vol. 52, 2006. 